\title{
An exit strategy for the tubercle bacillus?
}

\section{S. Hingley-Wilson and A. Lalvani}

Most studies on the host-pathogen interactions that occur between the residing infecting agent and its niche cell focus on the pathogenic manipulation of host cell entrance and intracellular propagation, but the timely and in-depth review by Kevin Hybiske and Richard S. Stephens (Exit strategies of intracellular pathogens. Nature Rev. Microbiol. 6, 99-110 (2008)) $)^{1}$ focuses on exit of the pathogen from its host cell. The timing and mode of action of the cellular egress by the bacteria may, as the authors denote, determine the efficacy of secondary infection and the immune response. In addition, it may affect the dissemination of the disease within the host and also the transmission between hosts. We understand that it was impossible for the reviewers to cover all intracellular infections that depend on extracellular egress. However, we also think it should be noted that cytolysis-dependent cellular egress has recently been described for the causative agent of tuberculosis, Mycobacterium tuberculosis, which remains the leading cause of bacterial mortality worldwide.

Indeed, the key mechanism involved in host cell egress by the tubercle bacilli forms the basis of the primary attenuating mutation (region of difference 1; RD1) of the vaccine strain Mycobacterium bovis bacille Calmette-Guérin (BCG).

Mycobacterial RD1 mutants exhibit a host cell lysis defect and exhibit reduced egress from host macrophages ${ }^{2-4}$. In the murine model of infection, RD1 was necessary to gain access to the deeper interstitial tissue of the lung and an M. tuberculosis RD1 mutant displayed both reduced disease progression and virulence ${ }^{4,5}$. Thus, in tuberculosis, RD1-mediated host cell exit is crucial both to cellular egress and to virulence. Interestingly, RD1 has recently been found to encode a novel type of secretion system and is responsible for both the production and secretion of a highly immunodominant virulence factor called early secreted antigenic target 6 (ESAT6) $^{4,6-8}$. We found that ESAT6 is itself capable of lysing an artificial membrane ${ }^{4}$, and it has since been reported that RD1mediated whole-cell lysis is induced through the depletion of intracellular ATP owing to lysis of the mitochondrial membrane ${ }^{3}$.

To conclude, we note that $M$. tuberculosis elicits cellular egress though RD1-mediated cytolysis, in a similar manner to many of the pathogens noted in the review, and that this is a requisite for disease progression and virulence. The mycobacterial mediators of cellular egress could be potential drug targets with which to halt dissemination and transmission of this global health threat. In addition, the benefits of understanding this potentially fundamental mechanism of disease progression include the development of much-needed improved strategies for the treatment and prevention of tuberculosis.

Tuberculosis Immunology Group, Department of Respiratory Medicine, National Heart \& Lung Institute, Wright Fleming, Institute of Infection \& Immunity. Imperial College London, Norfolk Place London W2 1PG, UK. Correspondence to S.H.-W. e-mail: s.hingley-wilson@imperial.ac.uk

1. Hybiske, K. \& Stephens, R. S. Exit strategies of intracellular pathogens. Nature Rev. Microbiol. 6, 99-110 (2008).

2. Volkman, H. E. et al. Tuberculous granuloma formation is enhanced by a mycobacterium virulence determinant. PLoS Biol. 2, e367 (2004).

3. Kaku, T., Kawamura, I., Uchiyama, R., Kurenuma, T. \& Mitsuyama, M. RD1 region in mycobacterial genome is involved in the induction of necrosis in infected RAW264 cells via mitochondrial membrane damage and ATP depletion. FEMS Microbiol. Lett. 274, 189-195 (2007).

4. Hsu, T. et al. The primary mechanism of attenuation of bacillus Calmette-Guerin is a loss of secreted lytic function required for invasion of lung interstitial tissue. Proc. Natl Acad. Sci. USA 100, 12420-12425 (2003).

5. Sherman, D. R. et al. Mycobacterium tuberculosis H37Rv: Delta RD1 is more virulent than M. bovis bacille Calmette-Guerin in long-term murine infection. J. Infect. Dis. 190, 123-126 (2004).

6. Stanley, S. A., Raghavan, S., Hwang, W. W. \& Cox, J. S. Acute infection and macrophage subversion by Mycobacterium tuberculosis require a specialized secretion system. Proc. Natl Acad. Sci. USA 100, 13001-13006 (2003).

7. Pym, A. S., Brodin, P., Brosch, R., Huerre, M. \& Cole, S. T. Loss of RD1 contributed to the attenuation of the live tuberculosis vaccines Mycobacterium bovis BCG and Mycobacterium microti. Mol. Microbiol. 46 709-717 (2002).

8. Guinn, K. M. et al. Individual RD1-region genes are required for export of ESAT-6/CFP-10 and for virulence of Mycobacterium tuberculosis. Mol. Microbiol. 51. 359-370 (2004). 